\title{
Computational Composites
}

\author{
Anna Vallgårda \\ IT University, Copenhagen \\ Rued Langgaards vej 7, 2300 Copenhagen, \\ Denmark \\ akav@itu.dk
}

\author{
Johan Redström \\ Center for Design Research, Royal Academy \\ of Fine Arts, School of Architecture, \\ Copenhagen, Denmark and \\ Interactive Institute, BOX 24081, SE 10450 \\ Stockholm, Sweden \\ johan.redstrom@tii.se
}

\begin{abstract}
Computational composite is introduced as a new type of composite material. Arguing that this is not just a metaphorical maneuver, we provide an analysis of computational technology as material in design, which shows how computers share important characteristics with other materials used in design and architecture. We argue that the notion of computational composites provides a precise understanding of the computer as material, and of how computations need to be combined with other materials to come to expression as material. Besides working as an analysis of computers from a designer's point of view, the notion of computational composites may also provide a link for computer science and human-computer interaction to an increasingly rapid development and use of new materials in design and architecture.
\end{abstract}

\section{Author Keywords}

Material, computational composites, interaction design, architecture, aesthetics

\section{ACM Classification Keywords \\ H.5 INFORMATION INTERFACES AND PRESENTA- TION, F.m MISCELLANEOUS}

\section{INTRODUCTION}

Throughout the last centuries, industrial design and architecture have been influenced, challenged and transformed by the development of new materials. The modernist design and architecture that came out of exploring new materials such as plywood, steel and reinforced concrete in the early 20th century is just one example. More recently, technological innovations such as smart materials and embedded computational resources have begun to influence design, in emerging areas such as smart textiles and interactive architecture.

Interestingly, also the context for human-computer interaction and interaction design is changing because of the avail-

(C) ACM, 2007. This is the author's version of the work. It is posted here by permission of ACM for your personal use. Not for redistribution. The definitive version was published in In Proceedings of the Conference on Human Factors in Computing Systems, San Jose, California, April 28 May 03, 2007 pp. 513-522 (http://doi.acm.org/10.1145/1240624.1240706) ability of such new materials. Areas such as ubiquitous computing, augmented reality, and physical computing, have made it evident that the personal computer is just one out of many possible ways in which we can design how humans interact with computers. Another illustration of this development is the increasing integration of interaction design and more general product design, i.e., how designing the interaction with computers becomes part of what it means to design products in general, be it in the shape of digital cameras, mobile phones, or electronic toys.

Issues related to materials are quite central to design. To shape the expressions and functions of their designs, designers need to know about the materials at hand. Or as Ezio Manzini states in the first sentence of his book The Material of Invention: "Every object made by man is the embodiment of what is at once thinkable and possible." Previously, what were 'thinkable and possible' were primarily linked to the direct experience with materials and manufacturing techniques; however, the contemporary invention of new materials and technologies make such an approach practically impossible [20]. Language therefore becomes a crucial part of a design process as a way to understand material and technological possibilities. The language of materials developed within science and engineering, however, does not automatically transfer into the realm of design. It needs to be appropriated to a design context where issues of expressiveness, aesthetics, and product manufacturing are more important than the technical properties.

Given the observation that knowledge of materials is essential to design practice and its development over time, some intriguing questions surface: In what ways can we consider computational technology as material? To what extent would such an understanding be based on computer science, and to what extent would new perspectives on this technology have to be developed to address the perspectives and issues designers deal with? How can we understand, and work with, computational technology in relation to other materials?

In what follows, we present an analysis of computers as material in design. To illustrate that this is not just a matter of metaphors, we discuss some central characteristics of materials and show how they also apply to computational technology. Further, to address issues related to misconceptions 
about computations being almost 'immaterial' and thus not really a material we can work with, we introduce the notion of computational composites as a way of understanding how computation comes to expression through an integration with other materials.

\section{BACKGROUND}

As discussed by Grudin already some 20 years ago [14], the use of computers in general, and the notion of the humancomputer interface in particular, is constantly evolving 'outwards'. Certainly, an example of such outward motion, the notion of Ubiquitous Computing now implies computers as part of, and embedded in, most kinds of everyday things and environments. The design of applications and interfaces has evolved towards a closer relation to other design areas also in terms of physical design. Approaches to interface design such as 'Tangible User Interfaces' can be said to point to an increasing interest in the combination of traditional design materials-and in physical objects as such-and computational technology. This stresses aspects such as the relation between information and its concrete presence to the user [29]. The development towards a closer relation between interface design and industrial product design, is quite visible in this area, with the approach developed by Djajadiningrat et. al (2004) as one illustration [6].

Outside the realm of human-computer interaction and interaction design, related developments are taking place. The use of computers in design is expanding beyond the use of computer-based tools to support design work, to become part of the designed things themselves. Textile designers explore the use of dynamic patterns made possible through 'smart textiles' instead of the static ones that traditional printing techniques afford. Architects show an increasing interest in the interactive properties of new technologies, and perhaps especially in the possibilities to program dynamic structures-be it lighting, sound, climate control, or surface expressions. Or just consider how communication and graphics design merge with architecture through the use of wall-sized displays on urban buildings running commercials or dynamic billboard ads.

As interactive technologies find their way into new areas of use, new intersections between areas of expertise are being opened. Inspiring new forms of collaboration, such new intersections often challenge the traditions and methodological approaches for everyone involved. A task for the research community is therefore to develop theoretical and methodological frameworks that can function as common language and grounds. It is our hope that $\mathrm{HCI} /$ interaction design and computer science will take an active role in developing these new intersections, and that the notion of computational composites could be a contribution to establishing such a common ground.

\section{Computers and Materials}

In examples such as the ones above, the boundaries between what we could refer to as human-computer interaction and other areas of design dealing with interactive technologies and 'smart materials' to some extent begin to dissolve. Cor- respondingly, the notion of a computer-be it a ubiquitous one-is not very illuminating, and instead we begin to use notions such as interactive products [25], digital artifacts [19], computational things [15], etc.

We propose that, as computational technology is no longer just a tool, it could instead be seen as a material-a material much like any other material we use to design things. One of the first proponents of a similar perspective was Seymour Papert: "In this project, the students built devices for measuring time using any materials they wished. Some used string and a metal weight to make a pendulum, some used plastic containers to dribble sand-and some used computers. Our central focus is this use of the computer as just another type of material." [23] "Just as pendulums, paints, clay, and so forth, can be "messed around with," so can computers. Many people associate computers with a rigid style of work, but this need not be the case." [23]

In another account of information technology as a material in interaction design, Löwgren and Stolterman suggest that it is a material without properties [18]. As a material without properties hardly qualifies as a material, what they hint at is that information technology seems to exist in-between the material and the immaterial with properties so flexible it almost can take on any form we want. Such a perspective, however, makes it difficult to understand how this material relates to other materials we use in design, as it almost seems to exist in isolation on its own premises.

In the work of Hallnäs and Redström [15], computational things are characterized by, on one hand, the temporal form that stems from computational processes and on the other hand the spatial form given to these processes by other materials with strong spatial form elements. A central example is the combination of computations and textiles, in which the dynamic properties of textiles are used to manifest temporal structures generated by computational processes (cf. also [27]). Here the computations comes to expression through the textile and together they form a new type of material. One suggestion, then, is that while computational technology is material (as distinct from being immaterial), it cannot really exist on its own in free form. To resolve its seemingly strange existence in-between the material and the immaterial, and its dependence on other materials for its presence, we propose thinking of it as a type of composite: that computational technology is a material, which we have to combine with other materials in order for it to become a material we can use in design practice.

Before we go any further into what exactly a computational composite is, we examine what composites generally are made of-the materials.

\section{WHAT IS A MATERIAL?}

The general concept of material is an ill-defined one even within material science. Generally, we can consider a material as a physical substance that shows specific properties for its kind. It can be understood as a substance with no specific 
form, which can be shaped and proportioned in volumes according to needs.

Materials can be divided into various kinds of groups, which exhibit similar reactions or properties. Examples of such groups are metallic and non-metallic, natural or artificial, brittle or ductile, translucent or opaque, and smart or ordinary. However, the meaningfulness of a grouping depends entirely on the point of view.

\section{The Sliding Scale of Materials}

There are a vast number of viewpoints from which one can contemplate materials and none of them is discrete. The scale of physical dimensions alone range from nanometers $\left(10^{-9} \mathrm{~m}\right)$ to kilometers, from molecules to steel wires [12]. Just as the state in which the material exists can vary from vapor to fluid to solid depending on the environment in which it is present.

Furthermore, the point of view changes with the purpose of engaging with the material, which often is correlated with the disciplinary background. Chemistry, physics, and engineering (even biology and geology in some cases) investigate and create materials per se, whereas for instance engineers, architects, industrial designers, and craftsmen of various kinds typically work with different applications of materials. However, the distinction between the disciplines is blurred and they often need to overlap for new materials to reach a market or for a market to demand new materials [12]. Thus, working with materials is inherently an interdisciplinary affair. Designers need to have an understanding of a material to make use of it and material scientists need to manage several levels of abstraction to study material properties in depth and to develop new useful ones. The following description of material will therefore employ different perspectives from different disciplines at different entries on the physical scale, to address aspects relevant to an account of computational composites.

\section{Structure and Material}

There is no clear distinction between what we would consider a structure and what we would consider a material. To some extent, it is a matter of how the material is approached. At a molecular scale, every material is a structure as the molecules form different kinds of patterns, such as: grids, rings, and double helixes, structures that are held together by various forms of energy. These structures are significant for the properties the material exhibit. Thus, on one level of abstraction every material can be seen as a structure.

Even at other levels of granularity can we find structural behavior in what we normally would be reluctant to call a material. Wood, for example, comprises a complex cellular structure, which resembles a collection of tiny drinking straws held together by chemical bonds. Wood grows by applying a new layer of 'drinking straws' right under the bark every year as long as it is growing [12]. Another example is a pile of brick which is clearly a structure, but cement, masonry and cast iron are considered materials even if they also are stronger in compression than they are in tension for the same reason: they are all full of cracks [12]. Thus, whether something is a material or a structure largely depends on the eye of the beholder.

\section{Material Surfaces}

Every material has a surface: an interface to the surroundings. It is typically the surface we encounter when we experience a material-its texture and color. The surface, however, can also play a significant role regarding the strength of material. Glass, for instance, is a material which in theory ought to be much stronger because of the strength of its chemical bonds, but our everyday experience shows a different result [12]. This is because of an inherent tendency in glass to cause tiny cracks in the surface. Cracks, which in turn causes a redistribution of tension resulting in a more fragile material than can be determined from the strength of its chemical bonds. Glass fibers, on the hand, can be made tremendously strong because it is easier to keep their surface smooth [12].

A surface of a material is largely dependent on the state it is in, whether a liquid or a solid substance. However, a surface is rarely in a completely stable state. Environmental conditions such as water, air, and sunlight, can cause oxidation leading to corrosion in metals or dissolution leading to corrosion of ceramics. Chemical reactions can cause the materials to change their properties, for instance their mechanical strength, their color, or their texture. Sometime this change is desired for aesthetic value, or even because the chemical reactions function as glue between two materials, but more often the surface needs to be treated to prevent alterations and thereby enable predictability.

\section{Material Properties}

As stated in the beginning of the section, we can generally view a material as a physical substance, which shows specific properties for its kind and which can be manipulated into something specific. A key word here is property. Besides availability and expense, properties are what make us choose one material over another.

Every material has a set of properties, which again varies based on the point of view. For a chemist, these are normally defined at a molecular level as to reflect potential chemical reactions. For an architect, on the other hand, such properties could include strength, optical properties, electrical properties, thermal properties and insulation, acoustic properties, deformations, deterioration, and appearance [8]. Thus, defining the properties of a given material is not just a matter of properly describing the given material, but about doing so with respect to a certain interest or perspective.

This relation between perspective and material properties is one reason descriptions and frameworks developed in, say computing science, does not automatically transfer into interaction design, or from engineering into architecture. Even if such frameworks are closely related and to some extent overlap, they still need to take offset in their focal point of interests to reflect the concerns at hand. 


\section{Composite Materials}

Combining two or more materials in composites is a way either to enhance a specific property or to introduce new combinations of properties in a material. This is often done with respect to strength, stiffness or toughness, but can also be done with respect to appearance, optical properties etc. [3]. A composite designed to improve material strength is usually made from a matrix and a fiber, where the three most common matrix types are polymer, metal and ceramic and fibers are usually made from ceramic such as glass or carbon, but can also be others kinds [17].

The properties of the individual constituents can give a clue about the properties of the composite. It is, however, complicated to predict the actual properties of a composite as its structure, e.g., the direction and shape of the fibers, and the interface between the constituents, will affect the result [17]. For instance, is anisotropy common for composite materials, meaning that they show differences in strength when measured in different directions-like an egg. As for the interface between the constituents, various chemical reactions can happen, which may affect the general properties. The exact constituents, the structure and the fabrication process to create a composite material are therefore often chosen with a specific application in mind [17].

One example of a composite material-or more correctly an alloy-is aluminum. Aluminum is refined from the naturally occurring bauxite to a state called pig-aluminum [7]. Although aluminum at this state has properties such as corrosion resistance and its light weight, it is a weak and seemingly useless material. Only through alloys with other metals does it receive the strength and flexible form it is commonly known for [7].

\section{Material and Product}

The distinction between a product and a material is also blurred: what can be a product to the material engineer might be a material to the designer. This is especially true when it comes to the highly engineered composite materials that enter today's market, such as glazing with integral sun control louvers or self-cleaning clay tiles [3]. Generally, however, for something to be considered a material, at some state at least, it must make sense to talk about shaping a chosen volume of it into something new-to create a new intent with it. To make a roof with the self-cleaning tile or to create a glass facade with the special glazing that allow for a seamless light control system. Therefore, even if the distinction between products and materials also is a question of viewpoint, not all products are materials.

This short introduction serves as the background on which we now wish to introduce the idea of computers as material in design.

\section{COMPUTERS AS MATERIAL}

Perceiving computers as a material is, as we said, more than a metaphorical maneuver. It is a question of accepting their similar characteristics as significant enough to hereafter work with the computer in the same manner we work with materials like aluminum or glass. This section will point to parallels between computers and other materials regarding their substance, their structure, their surface, and their complex states of being.

\section{The Substance of Computers}

The common reference to computational technology as 'information' technology holds connotations of it being something that deals with representations, signs, and meanings. This understanding has led to the perception that computers are more than electrified machines. On the level of abstraction on which we wish to encounter the computer, it does not deal with representations. Computational technology at this granularity handles only voltage according to stored sequences of (practically) discrete voltage levels and maybe input streams likewise of (practically) discrete voltage levels. They are, however, often called algorithms and data respectively. Every program has a physical manifestation when it enters the computer, even if the input device has representational keys that the programmer push to enter the program into the computer, a translation of the push of each key into voltage happens before it enters the computer.

Other labels commonly used when talking about computational technology are software and hardware, where software refers to programs that the computer executes, and hardware refers to the computer per se. This distinction tends to cause some confusion as software holds the meaning of both the abstract representation of a program, whether in binaries or in a higher level programming language, and the program in its physical manifestation, whether stored or in execution. The point of this is that both software and hardware are physical and can be manipulated as such, and that computers therefore can be seen as a substance albeit a rather complex one.

Furthermore, when working with substances it is meaningful to talk about dimensions; to have more of a computer means to have more processing power. That it is capable of treating more instructions per clock cycle than a less capable computer. A computer, however, is a device in the sense that it cannot physically be cut in half and still exist as a computer. Thus, where a traditional material's threshold for being diminished lies at the point where the molecular structure would no longer exist as a structure or where the fibers (e.g. in wood and textile)are no longer fibers, the threshold for the computer is where its structure needs to be intact. The computer's threshold, therefore, lies at a much higher point on the physical scale.

\section{The Structure of Computers}

Underneath the view of a computer as a substance, we find, as was the case with other materials, a complicated structure containing several different elements all which plays a significant role in the computational process. At one level of the physical scale these can be listed as a central processing unit (CPU), memory, buses and input and output devices (I/O devices). At a lower level, we would include the arithmetic logic unit (ALU), the registers, the central circuit, the clock etc. and at even finer granularity, the individual dig- 
ital circuits would be revealed. As with other materials the structure of the components is important for the overall properties of the computer. Another resemblance here is the role of energy in the structure. The state of computers can be found by examining the levels of voltage in the circuits and in a sense computers would not be computers if it were not for the voltage to constitute the processes. This is analogous to how energy in other materials holds the molecules together as a structure and thereby constitutes them as materials. With this comparison, we leave the structural view of the computer and instead we focus on the computer as a material.

\section{The Surface of Computers}

A computer's surface is the interface to the streams of discrete voltage levels, which exist within the input and output devices. The input device allows an exterior to provide new sequences of voltage levels, which can take the form of either data or algorithms, just as an output device delivers sequences of discrete voltage levels that can be interpreted as the result of computations. However, as the surface lies within the devices and not on either side we need to examine the devices more carefully.

Let us take the keyboard as a common example of an input device. The interface to the computer lies not in the keys per se, but in the discrete voltage levels resulting from an interpretation of the push of a specific key. Often, a keyboard is designed to enable a variety of different languages by enabling different encodings of every key. This is done within the computer, and the change is usually not visible on the keyboard itself. The interpretation of a key does not change, however, if the key changes color, form, or texture. Thus, we can find the surface of the computer exactly where we start to deal with discrete voltage levels. The same is true for the output device. Therefore, it becomes apparent that the surface of the computer needs to be coupled with other materials for us to better control what will happen with the computations. To directly insert meaningful sequences of discrete voltage levels is practically impossible for humans to accomplish.

If the input and output streams constitute the surface of the material then the input stream can be seen as the rear side and the output as the front. Even if they seem equal, they serve completely different purposes; where the output stream is the expressive side of the material, the input stream is the possibility of moderating the expression. An alternative would be to understand the computer as a self-contained system without an input or output as known from theory of computable functions of the original Turing machine $[11,21]$. However, such view leaves out the possibility of interaction and thus the ability to change the result of ongoing computational processes. In other words, this would make it less relevant as a material for design and thus for this endeavor.

\section{The Properties of Computers}

At one level of abstraction, the property of a computer can be seen as the computations (at a lower level it is a matter of strict causal processes treating sequences of discrete volt- age levels which then can be interpreted as computations). A property that is completely different from that of other materials, but a property no less. The computations allow for conditioned changes of whatever the output devices are combined with-pixels on a screen, shape of a wall, or patterns on a floor. However, in its raw form it holds only this abstract ability to compute, there are no mechanical properties of strength or stiffness to back it up, nor any acoustic, aesthetic or optical properties to speak of.

The strict causal process which constitutes the computations happens in a circuit board; however small and however shaped. The properties of a computer can therefore be compared with those of aluminum in its raw form, both holds potential for interesting and useful properties and both needs to undergo a treatment for the potential to be fulfilled. The computer needs to be combined to other materials for the computations to have an impact; thus we arrive at the notion of computational composites.

\section{COMPUTATIONAL COMPOSITES}

In the last section, we argued that a computer is a material, but also that its computational property, in its raw form, is difficult (if not impossible) to exploit. The conclusion was that the computer needed to be part of a composite with other materials to become useful in design. In this section, we will explore how the computations can come to use through different types of composites.

Composites are made to enhance specific properties or to introduce new properties by combining certain materials in certain ways. With computational composites, it is primarily a question of introducing new combinations of properties; namely, to introduce the ability of digital computations together with tensile properties, optical properties, electrical properties, thermal properties and insulation, acoustic properties, deformations, deterioration, appearance and so forth.

\section{The Property of Computational Composites}

Computations in this situation mean that events can happen conditioned by a set of data and an algorithm. Thus, it enables the other parts of the composite to behave beyond their otherwise normal behavior. Expressed more precisely, a computational composite can exist in a number of states (e.g. colors, shapes, or positions). Whenever a set of conditions is met, a transition towards a new state is begun. The conditions and their fulfillment are controlled or computed in one of three ways:

1. With both algorithm(s) and data set predetermined.

2. With only the algorithm(s) predetermined, and the data set collected dynamically.

3. With a predetermined offset of conditions that changes dynamically, for instance based on a dynamically collected set of data.

An algorithm or a data set can also express approximated randomness and thus create a seemingly chaotic behavior in the composite when that is desired. 
The computations enable not just flexibility and change in the material expression, but they enable controlled transitions between states in the composite material. The control, the transitions and the states are the key aspects to take into considerations when composing a computational composite. The type of design choices to be made on all three accounts entails that it often will be necessary to compose computational composites with a specific purpose in mind, just as is true for other material composites. The control itself, for instance, must be a meticulously designed as a series of controlled transitions between states inside the computer of which only few become output and thus result in transitions between states in the composite material.

\section{The Structure of Computational Composites}

To honor the possibilities of computations, the composite need to be able to make the transitions as well as to stay in the chosen states. Thus, not only must the controls be carefully designed, but the rest of the composite must also match the controls. To assist the analysis of the structure of computational composites we now introduce two metaphors for the surface of the material: the front and the rear.

The front of the computational composite needs to be designed such that a computed result (the output) translates to a transition towards a new state in the composite. In dual or multiple state materials, such as shape memory alloys, nickel chromium wires, or liquid crystal displays, this can be done simply by letting the computations control the flow of electrical current, i.e. making electrical current function as 'glue' between the elements. Materials with only one state of being, such as steel or concrete, need more than electrical current to honor the possibilities of the computations. Single state materials can exploit the computations only by being in a structure with an additional actuator (e.g. a motor, or heat sensitive color) that can utilize the electrical current. All these translations combined can be seen as the 'glue' between the different materials in the composite.

The rear side of the composite is the access to the input stream as described above. The input stream consists of what we refer to as algorithms and data and constitutes the control of the composite. As such, it plays a central role in the composite material and the possible product to be made of it. The rear side can be designed such that it is constantly accessible throughout the life of the material (or product), or the algorithms can either be formed and frozen during the design of the material or during the design of the product. The same holds for the data set.

Dynamic input can happen through sensors or through connections with other computers. A sensor usually detects input by measuring: change of light, change of scenery, change of temperature, or change of pressure. The measurements then need to be translated into discrete voltage levels to enter the computer. This translation can happen in various ways: if it is a matter of a two state input, the translation is straightforward; otherwise, methods, such as a register over sequences of discrete voltage levels with the direct input from the sensor as a key to look it up, can be utilized.
Generally, as computers can be integrated in networks so can computational composites and thereby form large structures of the same material.

This leads to another aspect of the composite composition, as the computer, including the input and output streams, can exist in the composite in various degrees of integration. The degree of integration depends largely on the purpose of the composite; if the complication of computations entails a computer of a certain size, seamless integration can be impossible; or if dynamic input is needed, the input stream could require a device that resembled a tool more than a rear side of a material. More explicitly, the degree of integration depends on: the type of input needed, the size of the computer, the access to power supply or battery lifetime, whether it is a standalone computer or it is in a network, either in a server/client architecture or a distributed one; but it also depends on the other type of materials used, and the states which they need to assume. However, as long as the computations are utilized to control transitions between states in the composite material, it is a computational composite.

\section{EXAMPLES OF COMPUTATIONAL COMPOSITES}

In this section, we will explore examples of computational composites to render the concept more tangible. The first examples are different types of displays. Together they hint at the vast potential of embedding computations for both practical and aesthetic purposes. The last examples introduce flexible form as another mode of computational expression. Flexible form can for instance be used to alter the size of a given space by adjusting to specific purposes within that space.

Besides being examples of new kinds of materials, they are also illustrations of successful interdisciplinary collaboration between research, design, craft, and art. As such, they illustrate what working with computational composites might be like also in terms of the interdisciplinary approach needed to develop them.

\section{Computational Textiles}

The e-broidery project [24] propose several examples of computational textiles in which they use materials for the computer's electrical circuits that correspond to the flexibility, durability, wash-ability, and conformity of textiles. Thus, the computer is literally woven into the fabric. Metallic silk organza, for instance, can be used to create a conductive layer in which each thread can function as an individual ribbon cable because of the woven structure of the fabric [24]. The organza, working as a conductive layer, can be attached to other fabrics to insulate it from the surroundings and from folding. Another example of such a material is the conductive yarns made of stainless steel, which can replace any traditional wiring to and from the microprocessor [24].

In a later and more developed design called the Electronic Plaid $^{\mathrm{TM}}$ made by International Fashion Machines (see fig 1), a computational textile enables controlled change of color using the same principal of woven electronic circuits combined with color change inks and drive electronics [22]. Pre- 


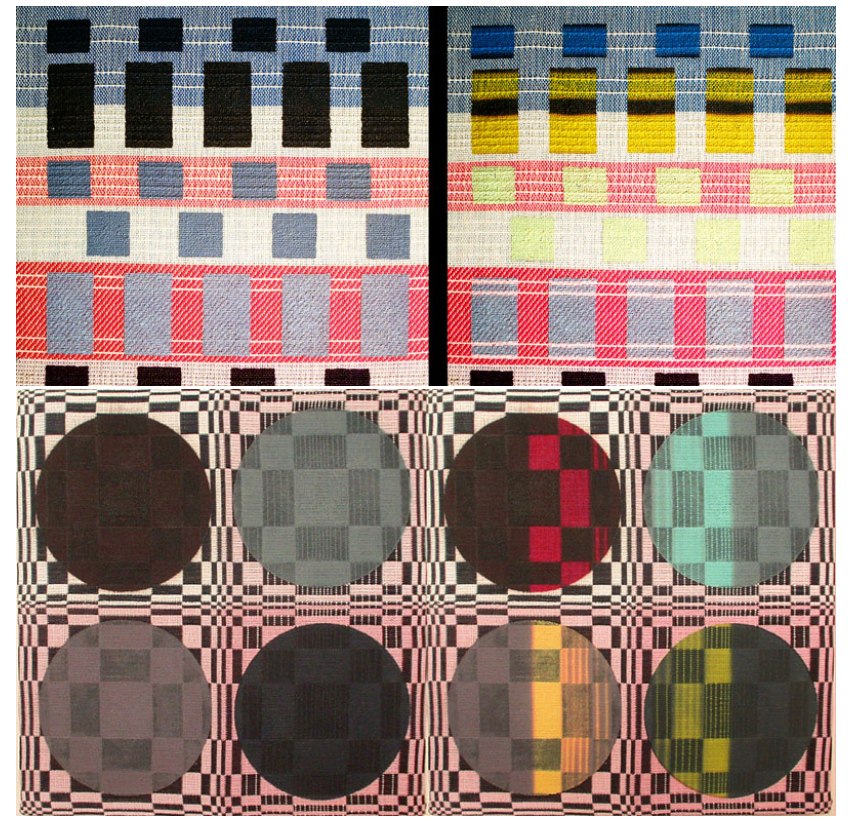

Figure 1. Examples of computational textile, courtesy of IFM [22]

sumably, a computer controls the electrical current in the circuits, which in turn initiate a color change (state change) under the right conditions. Thus, the electrical current is the 'glue' of the composite and the color changing ink is the actuator. The plaids are programmed in modules of eight pixels made of four to eight different electronic yarns that create the pattern [22].

This material is plastic, flexible, soft, and decorative, but it is sensitive to environmental conditions such as sunlight and water, and shows little mechanical strength. Thus, it is primarily suited for indoor wall displays or decorations and maybe even furniture [22]. The variety of dynamic expressions the material can display is limited to the four to eight different yarns per pixel, which can change between just a few colors. The material as available from International Fashion Machine is pre-programmed meaning that both algorithm and data input are determined before use. However, the design of the interaction with the textile needed not be determined beforehand it seems feasible to integrate automatic dynamic data collection for example various types of environmental sensors. This open-ended approach leaves for instance room for an interaction designer to decide how the textile is to be used.

\section{Computational Concrete}

Another example that relies on a similar type of composite structure is the Chronos Chromos Concrete (see fig. 2). This computational concrete is a composite material that holds the properties of ordinary concrete and still is able to dynamically display text or other patterns through color change [9]. The material behind the color change is thermodynamic ink, which is blended into the concrete. Beneath the concrete surface are mounted nickel chromium wires that heat up when

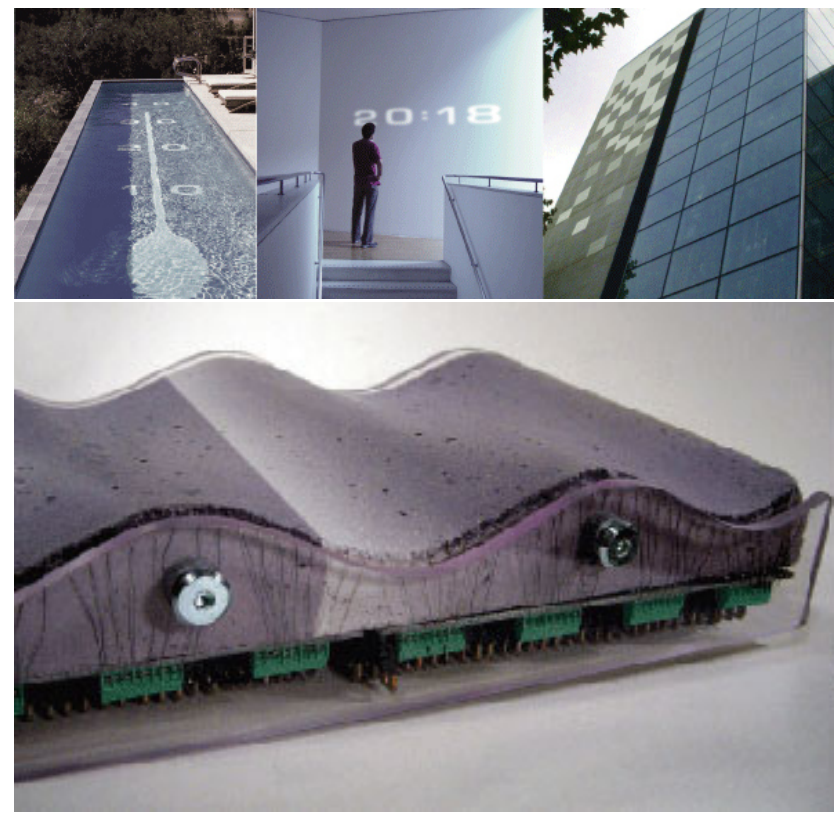

Figure 2. Example of computational concrete, courtesy of Glaister, Mehin, and Rosen [9]

electric current is passed through them. Then, when a certain temperature is reached in the concrete, it causes its color to change (state change); a process that takes at the minimum of five seconds [10]. As was the case with computational textile, the electrical current plays the role of 'glue.' The material is mechanically robust and thus suited for large-scale architectural installations. Its display dynamics is, however, restricted within the five seconds, and the patterns seem to lack sharpness up close. Even so, the developing potential seems extraordinary and the prototypes to date developed show concrete examples of a computational composite.

\section{Computational Tensegrity}

There are several projects where the walls or the whole building structure can move or change shape and thus either expand or diminish spaces on either side. Such ideas existed even before the digital computer was invented. Cedric Price's vision of the Fun Palace or Rogers and Piano's plans for the Centre Pompidou all build on ways of utilizing machines to create dynamic spaces. In less spectacular projects, but no less novel, several experimental architects today explore the possibilities in computations to enable dynamic spaces. Here, we take a closer look at two examples of how this can be achieved.

oframBFRA (The Office For Robotic Architectural Media and The Bureau For Responsive Architecture) has created a full-scale prototype of a tensegrity structure, which can be used as a responsive wall (see fig. 3) [5]. A tensegrity is a skeleton structure that consists of members in continuous tension and members in discontinuous compression. They are interconnected in a way that allows each member to contribute to a self-stressing structure. In the oframBFRA variation the tensegrity is a repeated module that consists of three 


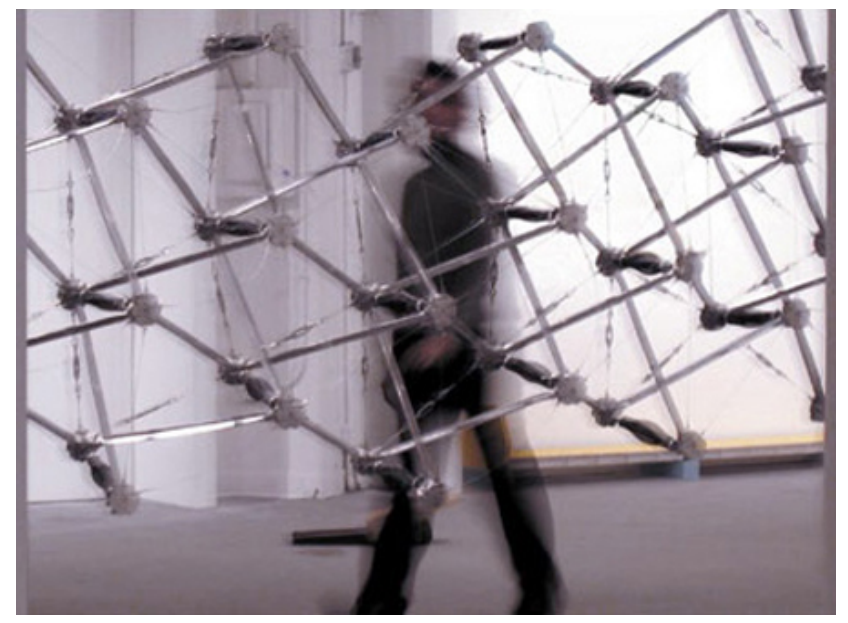

Figure 3. A prototype of an actuated tensegrity structure, courtesy of oframBFRA [5].

compression members which meet in a tripod formation that is held together by tension cables [5]. This forms a structure that can be subjected to alteration in tensions within each module causing the local rigidity to change and thus induce the entire structure to change shape [5]. Thus, by introducing an actuator controlled by computations on the apex of each module the tensegrity can perform controlled transformations between a wide number of states [5]. This is an example of a composite material where the complexity of the structure within the material resembles that of wood, but as it is presented by oframBFRA, to a much larger physical scale. The tensegrity can be covered by membranes, which enables it to form shelter and separate spaces. The dimension and control of the tensegrity are not a given; it can be scaled to suit different needs, and the controls can equally be designed for those purposes. oframBFRA suggests that the controls should rely on predetermined algorithms and let the data for the algorithms be dynamically collected through sensors that detect changes in the immediate environment.

\section{Moving Structure}

In Moving Structure [16], the architect Pavel Hladik exploits combinations of Teflon foils and the two states available in shape memory alloys (SMA) NiTiCu (See fig. 4). A SMA change shape according to temperature with a straight shape in its cold form to a bended shape (up to 5\%) when it is heated. The shape for the hot state is created during construction and remembered when the SMA is later reheated. The SMA's transition state lies at around $30^{\circ} \mathrm{C}$ but can be protected from the environment through a heat protection layer and thus become more controllable [16]. In Moving Structure, Hladik forms structures of spiral formed SMAs, Teflon foil, and lightweight heat emitting conductive fibers that coupled to a computer become a computational composite. The Moving Structure forms a material suitable for walls in many different situations [16]. As with the tensegrity structure, the algorithms and data sets are not necessarily predetermined and can therefore be designed to the chosen purpose of the material.

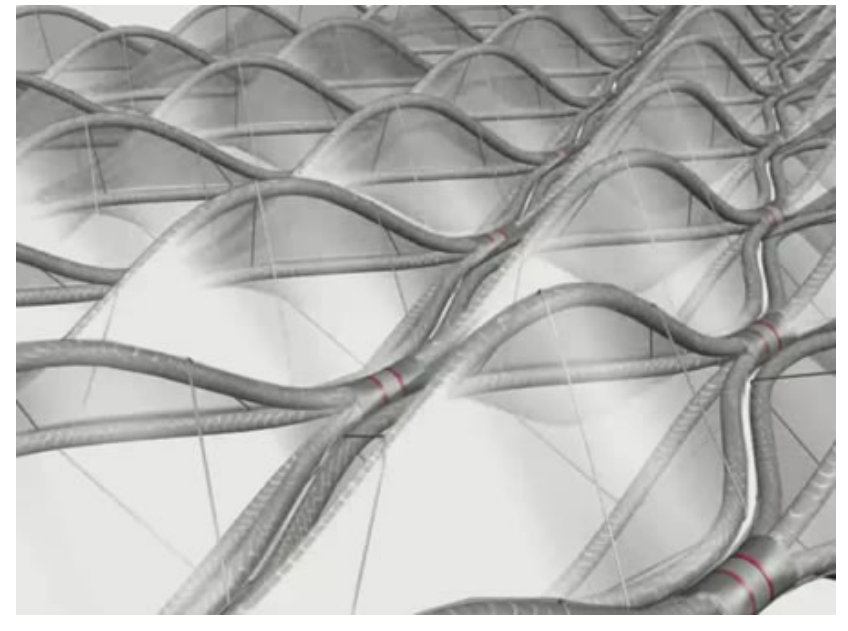

Figure 4. The SMA work as an actuator changing the shape of the entire material, courtesy of Pavel Hladik [16].

\section{Further Examples}

These were just a few illustrations of what computational composites might be like, and many more are available. The area of computationally enhanced textiles is, for instance, rapidly growing (cf. $[2,27]$ ) just as there are a wide variety of displays which utilize different materials to express the result of computations. One such example is the Pixel Skin 02, designed by Orangevoid, which also utilizes the two state material SMA to achieve changes in a surface [1]. Another example is the Wooden Mirror by Daniel Rozin. It utilizes wood which does not have an inherent actuator to ensure transitions between states, but by cutting it into pieces and appending a motor as actuator he achieves a similar effect as the Pixel Skin 02 [28]. The HypoSurface [13] integrates the display with a shape changing surface and in that endeavor rely on an even more complicated set of actuators which push it towards the boundary of being a material, however, it still poses material like properties. Lucy Bullivant's Responsive Environments [4] holds examples of even more moving, interacting and responsive materials and products.

The list seems endless, but most of these examples are still at a stage of research and need more refinement to become robust and reliable materials. Many of them have been designed as one-off installations or art pieces and not really as material to be produced in larger quantities. There exists, however, a few deployed examples such as the Diaphragm of L'Institut du Monde Arabe in Paris by Jean Nouvel, where he use irises to let different amounts of light through the windows based on light-sensor input.

\section{DISCUSSION}

Based on the observation that knowledge of materials is essential for design practice we raised a series of questions in the introduction: In what ways can we consider computational technology as material? To what extent would such an understanding be based on computer science, and to what extent would new perspectives on this technology have to be 
developed to address the perspectives and issues designers deal with? How can we understand, and work with, computational technology in relation to other materials? Based on the analysis presented, we now conclude the paper with a discussion of these questions. We will also relate them to existing design practices and technological research traditions, to point to future work in this interdisciplinary field.

The analysis of computational composites as presented in sections 'Computers as Material' and 'Computational Composites' provides our suggestions as to how computers can be considered a material: that computers can be understood as materials in the traditional sense and that computer's properties only become available when existing in a composite with at least one other material. We also argued that in order for design practice to come to grips with computational technology, we need to develop our frameworks beyond the one we now find in computer science, as it is necessary to deploy a new perspective on the technology. Given the development processes toward using other materials in design, the need for such re-appropriation is not unexpected-in fact, it is what happens most of the time in the interdisciplinary context of material development.

\section{Language and Framework}

When studying development and use of more traditional materials such as wood, steel, or aluminum it becomes clear that different types of access to a material are necessary because a chemist's approach to any given material is different than that of an architect. A difference caused by the need of minimizing the level of complexity. The matters focal to the chemist, such as the molecular structure and responsiveness with other chemicals, are circumferential to the architect, just as aesthetics and maintenance are to the chemist. If they were both to focus on every matter concerning the material, it would be hard to talk of a focus. The consequence of this is that every level of interest concerning the material needs to have a framework of concepts at their disposal. These frameworks need not be discrete and isolated from one another, but they must contain concepts that support the different perspectives. We find the same division of perspectives necessary for computer technology to propagate beyond computer science and into design or architecture. Therefore, it is necessary to try to conceptualize different perspectives of computational technology and thus complement existing computer science frameworks. HCI and interaction design have the potential to play an important role in this development.

\section{Design of Materials}

Our notion of computational composites is a framework that could allow for design practices in industrial design and architecture to work with the material on a different level of abstraction; an abstraction that we believe still accommodates the complexity needed for designers to propose feasible designs containing computer technology. The abstraction does not, however, remove all complexity of the material and the question remains: How can a designer use a material that is so complex it needs to be designed first? As the examples of computational composites illustrate, there seems to be a continuum of development and use ranging from the design of a new 'raw' composite material to be used in ways yet to be determined, to the development of a certain application, product, or environment. Again, this situation is not unlike what we find in other areas of design. Textiles may serve as an example. At one end of the scale, textile engineers research and develop fibers, materials and production techniques. Based on this, textile designers create textiles for designers to utilize for clothes, furniture, or art. The development of textile artifacts happens in layers that, though certainly intimately connected, do deal with different sets of issues. Even within design and development of traditional computers we find a division of labor and interests. In the case of computational composites, however, the matter to be designed and developed is different from a traditional computer; it demands more of a material science perspective on the result than a traditional computer science perspective. Furthermore, interaction designers, architects and artists are bound to play a much larger role developing the material on its way to become a product.

Developing computational composites is not a matter of simple distinctions between technology and its application it is about rather intricate and highly developed layers inbetween. With respect to interaction design, this opens some interesting perspectives. For instance, there could be a choice between working with the development of new materials (as in how the textile designer creates new fabrics we all can buy and make new curtains from), and working with finalized products based on such materials (as in how the fashion designer makes garments we wear). We might even say that traces of such layers exist in previous developments of human-computer interaction, with the notion of end-user programming, or in the interest in DIY kits for ubiquitous computing applications. We even find traces of similar layers in more ordinary computer use. Users of desktop computers differ significantly when it comes to how 'deep' their customizations of the machine is: from just filling it with personal content such as documents and images, to extensive personal modifications of both software and hardware.

\section{A Non-Functionality Perspective}

Working with computational materials in the ways discussed in this paper, could also be a complement to existing approaches to interaction design. It does not depend on specific notions of functionality in the same ways as the development of 'applications' does [26]. Instead, it centers on notions such as material properties, which represent a rather different starting point for explorations of new possibilitiesespecially so when it comes to the increasing collaboration between designers of different domains on how computing can be utilized.

Thus, with the notion of computational composites we are not only seeking to provide a material view on computation that various designers could benefit from - we also propose a material science perspective on computer science that could open up for new forms of collaboration between computer science and architecture, human-computer interaction and design 


\section{ACKNOWLEDGMENTS}

We thank Kjeld Schmidt for useful comments and discussions and Ruairi Glynn with his interactivearchitecture.org for being a useful source of examples. We also thank the program committee for their discussion of the paper and the reviewers for their many useful comments.

\section{REFERENCES}

1. Anshuman, S. Pixelskin02: www.orangevoid.org.uk. Webpage (March 2 2007).

2. Braddock-Clarke, S. E. and O'Mahony, M. TECHNO TEXTILES 2 Revolutionary Fabrics for Fashion and Design. Thames and Hudson, New York, NY, 2006.

3. Brownell, B. Transmaterial - A Catalouge of Matreials that Redefine Our Physical Environment. Princeton Architectural Press, New York, NY, 2006.

4. Bullivant, L. Responsive Environments - Architecture, Art and Design. V\&A Contemporary, London, UK, 2006

5. d'Estrée Sterk, T. Using actuated tensegrity structures to produce a responsive architecture. In Annual Conference of the Association for Computeraided Design in Architecture. ACADIA, Indianapolis, Indiana, 2003, 25-93.

6. Djajadiningrat, T., Wensveen, S., Frens, J., and Overbeeke, K. Tangible products: Redressing the balance between appearance and action. Personal and Ubiquitous Computing, 8, 5 (2004), 294 - 309.

7. Doordan, D. P. Promoting aluminium: Designers and the american aluminium industry. Design Issues, 9, 2 (1993), 44-55.

8. Everett, A. Materials. Pearson Education, Harlow, England, 1994, fifth edition.

9. Glaister, C., Mehin, A., and Rosen, T. Chronos chromos concrete: www.chromastone.com. Webpage (January 14 2007).

10. Glynn, R. Interactive architecture: www.interactivearchitecture.org/chronos-chromosconcrete.html. Webpage (January 14 2007).

11. Goldin, D., Smolka, S., Attie, P., and Sonderegger, E. Turing machines, transition systems, and interaction. Information and Computation, 194, 2 (2004), 101-128.

12. Gordon, J. E. The New Science of Strong Materials - or why you don't fall through the floor. Princeton University Press, Princeton, UK, 2006, second edition.

13. Goulthorpe, M., Shpiner, S., and Allen, C. W. Hyposurface: http://www.hyposurface.org/. Webpage (January 14 2007).

14. Grudin, J. Interface: an evolving concept. Communications of the ACM, 36, 4 (1993), 110-119.
15. Hallnäs, L. and Redström, J. Interaction Design: Foundations, Experiments. The Swedish School of Textiles University College of Borås and Interactive Institute, Borås, Sweden, 2006.

16. Hladík, P. Moving structure: http://www.movingstructure.info. Webpage (January 14 2007).

17. Hull, D. and Clyne, T. W. An Introduction to Composite Materials. Cambridge University Press, Cambridge, UK, 1996, 2nd edition.

18. Löwgren, J. and Stolterman, E. Design av informations-teknologi: Materialet utan egenskaper. Studentlitteratur, Lund, Sweden, 1998.

19. Löwgren, J. and Stolterman, E. Thoughtful interaction design - a design perspective on information technology. MIT Press, Cambridge, MA, 2004.

20. Manzini, E. The Material of Invention - Materials and Design. MIT Press, Cambridge, MA, 1989.

21. Milner, R. Elements of interaction: Turing award lecture. Communications of the ACM, 36, 1 (1993), $78-89$.

22. Orth, M. et al. International fashion machines (ifm): www.ifmachines.com/eplaid.html. Webpage (January 14 2007).

23. Papert, S. Computer as material: Messing about with time. Teachers College Record, 89, 3 (1988), (http://www.papert.org/works.html, Januar 14 2007).

24. Post, E. R., Orth, M., Russo, P. R., and Gershenfield, N. E-broidery: Design and fabrication of textile-based computing. IBM Systems Journal, 39, 3 and 4 (2000), $840-860$

25. Preece, J., Rogers, Y., and Sharp, H. Interaction Design; Beyond Human-Computer Interaction. Wiley \& Sons, Wokingham, England, 2002.

26. Redström, J. On technology as material in design. In A.-M. Willis, ed., Design Philosophy Papers: Collection Two, Team D/E/S Publications, Ravensbourne, Australia, 2005. 31-42.

27. Redström, M., Redström, J., and Mazé, R., eds. IT+Textiles. IT Press, Edita Publishing, Helsinki, Finland, 2005.

28. Rozin, D. Wooden mirror: www.smoothware.com/danny/woodenmirror.html. Webpage (January 14 2007).

29. Wisneski, C., Ishii, H., Dahley, A., Gorbet, M., Brave, S., Ullmer, B., and Yarin, P. Ambient displays: Turning architectural space into an interface between people and digital information. In N. A. Streitz, S. Konomi, and H.-J. Burkhardt, eds., Cooperative Buildings Integrating Information, Organization, and Architecture. Springer-Verlag, Darmstadt, Germany, 1998, 22-32. 